\title{
Analysis of the impact of construction and operation of pumped-storage power stations on grid companies and the formulation of electricity prices
}

\author{
Ming Gao ${ }^{1, *}$, Jiayu Bian $^{1}$, Shoutao Tian ${ }^{1}$, Jing Tan $^{1}$, and Lufeng Chen ${ }^{1}$ \\ ${ }^{1}$ Economic and Technological Research Institute of State Grid Xinjiang Electric Power Co., Ltd, \\ China
}

\begin{abstract}
The pumped storage power station is flexible to start, can realize effective storage of electric energy, and has superior peak and frequency modulation effects, which is beneficial to provide comprehensive benefits of the power system. But at present, the construction of pumped-storage power plants has obviously exceeded the speed of policy and market development, resulting in problems in pumped-storage power station operation and cost reduction. Aiming at this problem, this paper further expounds the influence of the construction and operation of pumped storage power station on the electricity price of power grid companies. The revenue of pumped storage power station mainly comes from the auxiliary service market. In China, the peak regulation effect of pumped storage power station is significant and the revenue is considerable. Therefore, this paper studies the formulation of time-of-use price and subsection price of pumped storage power station.
\end{abstract}

\section{Problems and difficulties faced by the construction and operation of pumped storage power stations}

\subsection{Problems faced by the construction and operation of pumped storage power stations}

The site selection of pumped storage stations is limited by external environmental factors, with high requirements on water head and geology, and limited sites with good geographical location and natural conditions. Therefore, the role of pumped storage in delaying power grid upgrading cannot be flexibly improved. The main role of pumped storage is peak shaving. But whether the main body of pumped storage is a grid company or a non-grid company, its benefits are difficult to recover.

\footnotetext{
* Corresponding author: zhangshuting0519@,163.com
} 


\subsection{Difficulties in diverting costs for pumped storage power plants}

The main body of the pumped storage power station is the power grid enterprise, and when the operation mode is unified operation of the power grid, the power station is the effective asset of the power grid. The price of pumped-storage electricity can be included in the transmission and distribution price for cost relief. The main operation strategy is to perform peak shaving according to dispatch orders. However, the construction costs of different pumped storage power stations are different. First of all, in order to minimize the cost of the whole system, the pumped storage power station is often not called when the peak load of thermal power units is abundant[1], Secondly, the government clearly put forward the task of lowering general industrial and commercial electricity prices. The sales price of electricity also does not have the basis to ease the cost of pumped storage power stations. The return on investment cannot be guaranteed, and the benefits of pumped storage power stations are often difficult to recover.

The main body of pumped storage power station is non-power grid enterprise, and the operation mode is power grid leasing. The power station does not belong to the effective assets of power grid. The benefits of pumped storage are often recovered through the electric energy market and the auxiliary service market, mainly in the auxiliary service market. Pumped-storage power stations buy electricity at low prices when the power grid is at a low price, and arrange to participate in the two markets to get the maximum benefit. However, the current ancillary service market is not perfect and it is difficult to guarantee the recovery of revenue.

Pumped-storage power station is mainly a non-power grid enterprise, and its operation mode is independent, and its cost recovery mode is the same as that of power grid leasing.

\section{The impact of the construction of pumped storage power stations on the electricity prices of grid companies}

Pumped storage power station is invested by the power grid company. If it is not included in the electricity price of transmission and distribution, its construction has no influence on the electricity price of the power grid company. Taking into account the electricity transmission and distribution price, the cost of the power grid company becomes higher, so the power grid company needs to make reasonable arrangement and dispatch for benefit recovery[2]. Under the current policy, power grid companies buy electricity from power generation groups with transparent prices. The benchmark electricity prices set by the state are on the grid. Electricity sales prices are implemented in accordance with eight types of sales electricity prices. Pumped storage electricity prices have no effect on electricity prices on both sides, but may have benefits.

\section{Formulation of time-of-use electricity prices for pumped storage power stations}

This paper uses the long-term marginal cost method to calculate the time-of-use electricity price of pumped storage power stations. In the practical marginal cost method, the idea of capacity cost allocation is to use part of the basic electricity price as the basic electricity price, which has nothing to do with the generation period, and the remaining capacity cost is allocated to the peak, flat, and valley periods as the electricity capacity cost. The following research basic electricity price and peak, flat, valley three time period electricity price calculation method. 


\subsection{Basic electricity price}

The existing method is to assume that the idle cost of unit capacity is the same as the operating cost. Considering that the capacity cost allocated per unit hour during unit operation should be greater than the capacity cost allocated per unit hour during idle time, Multiply by system $x$ greater than 1 before the annual average daily load rate of the unit[3]. The basic electricity price calculation formula is as follows:

$$
P_{\mathrm{b}}=M C_{f} \cdot(1-\theta \cdot x)
$$

where, $M C_{f}$ is peak load capacity electricity price; $\theta$ is the annual average daily load rate of the nuit.

\subsection{Electricity price per degree}

\subsubsection{Peak charge electricity price}

The peak charge price is composed of three parts: the marginal electricity cost, the change value of the variable operating cost, and the apportionment of the plating capacity cost. Considering that $F_{s}$ is the change in operating cost caused by the increase in the marginal capacity per kilowatt during the peak load period, it is all included in the peak load price.

$$
P_{f}=M C_{v f} \pm F_{s} / T_{f}+M C_{f} \cdot \theta \cdot k / T_{f}
$$

where, $M C_{v f}$ is the peak charge electricity price; $F_{s}$ is the charge in fuel cost after adding unit kilowatt capacity to the system; $k$ is the apportionment of capacitance cost in peak load period; $T_{f}$ is the annual number of hours in peak load period.

\subsubsection{Flat charge of electricity}

The flat charge price is composed of marginal cost of electric quantity and cost allocation of electric capacity.

$$
P_{p}=M C_{v p}+M C_{f} \cdot \theta \cdot(1-k) / T_{p}
$$

where, $M C_{v p}$ is the marginal cost of electric quantity in the flat load period; $T_{p}$ is the annual number of hours in the average load period; Other variables have the same meaning.

\subsubsection{Valley load price}

$$
P_{g}=M C_{v g}
$$

(4)

where, $M C_{v g}$ is the marginal power cost in valley charge period. 


\section{Establishment of section electricity price for pumped storage power station}

In the electricity market, generators' quotations should be based on the cost of electricity generation, and they should study the method of setting electricity prices in stages under competitive bidding. Figure 1 shows the segmented bidding market model[4]. From zero load to the highest load, it is divided into 1 sections, and the marginal cost method is used to calculate the electricity price of each section.

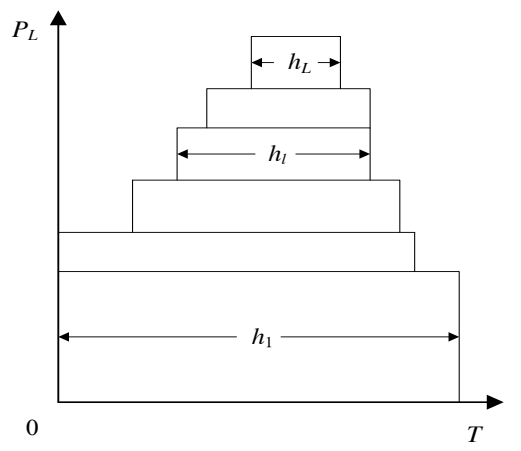

Fig. 1. Segment bidding market model.

It is assumed that the pumped stoage unit adopts continuous operation mode to bid for the first stage of load. The daily duration of this stage of load is $K_{i}$, and the unit adopts pressure load operation during the non-operation period[5].

1) Electricity price in the base charge segment

The first section is the base load section, and the load duration is 24 hours. The unit power cost is composed of fixed cost, variable cost and apportioned start-up cost. According to the power production characteristics, the units located at the base load shall be in continuous operation. The determination of electricity price in the base charge segment is divided into the following three steps:

i.Based on the existing operation data, the continuous operation time of the base machine sister in the first stage and the continuous operation time in subsequent stages were calculated, and the cumulative frequency curve of the continuous operation time after the unit was started up was drawn.

ii.Select the guarantee rate (such as $95 \%$ ) to determine the continuous running time of a startup;

iii.The startup cost is divided equally among the running time and divided among the capacity segments. This can effectively avoid risk, and the price curve rises segment by segment.

The formula for calculating the electricity price in the base charge segment is as follows:

$$
b_{l, k}=\frac{C V_{l, k}}{24}+\frac{S_{k}}{T_{l, k, p} P_{l, k}}+C E_{l, k}
$$

where, $b_{l, k}$ is unit power cost of unit $\mathrm{k}$ the base charge segment; $C V_{l, k}$ is the marginal capacity cost of unit k in the base load segment; $C E_{l, k}$ is marginal power cost; $S_{k}$ for the start-up and shutdown cost of unit k; $T_{l, k, p}$ are the operating time of unit $\mathrm{k}$ in the base charge segment and the subsequent segment when the guarantee rate is $P ; P_{l, k}$ is the force of unit k in the base load segment.

2) Electricity price in subsequent stages 
The load segment above the base load segment is called the load subsequent segment and is denoted as $1,1 \neq 1$. The unit marginal cost of electric power in section $L$ consists of the following parts:

Marginal cost per unit of power. $C V_{l, k}$ and $C E_{l, k}$;

Starting and stopping costs apportioned in this paragraph. There are two cases:

Scenario 1: The base load segment is successfully bid.

Set the generating capacity of unit $\mathrm{k}$ in stage 1 as $P_{l, k}$, then the apportionment of downtime costs is $S_{k} /\left(T_{l, k, p} P_{l, k}\right)$;

Case 2: the preceding paragraph is successfully bid, but the preceding paragraph is not a base paragraph, or the preceding paragraph is not successfully bid, this paragraph is the first paragraph of the bid. The unit adopts daily open and shut down operation mode to meet the requirements of minimum continuous operation time and minimum continuous shutdown time. Set $T_{l, k, p}$ as the continuous operating time of unit $\mathrm{k}$ in a day when the guarantee rate is $\mathrm{P}$, then the apporated downtime cost is $S_{k} /\left(T_{l, k, p} P_{l, k}\right)$;

3) The added value of power generation costs of the preceding extension

If the load in this section is not the first load borne by unit $\mathrm{k}$, the increase of fuel cost and operation and maintenance cost of the front continuation section (the first section from the front to the load borne by unit k) should be taken into account when calculating the segmentbased electricity price[6].

\section{Conclusion}

In recent years, the momentum of energy storage has risen sharply, and society's attention to pumped storage power plants has also increased. However, the cost recovery of pumped storage power plants currently requires support from policies and external conditions such as market maturity. This article discusses pumped storage time sharing. And the method of formulating the segmented electricity price will provide a theoretical basis for the quotation of pumped storage power stations when the market matures in the future.

\section{References}

1. J.zou, X.lai, PROC CHIN SOC ELECTRICAL ENG, 02 564(2017)

2. X.wen, S.zhang, et al, POWER SYS TECHNO, 06 487(2018)

3. L.zhang, H.ye, et al, PROC CHIN SOC ELECTRICAL ENG, 14 92(2017)

4. S.liu, Z.ye, et al, PROC CHIN SOC ELECTRICAL ENG, 24 58(2017)

5. Y.han, W.tian, et al, POWER SYS TECHNO, 07175 (2011)

6. Z.zhao, D.zhang, et al, POWER SYS TECHNO, 02 94(2016) 